УДК 621.039 .514

DOI: $10.32326 / 1814-9146-2020-82-3-339-352$

ЧИСЛЕННОЕ МОДЕЛИРОВАНИЕ НЕСТАЦИОНАРНЫХ ПРОЦЕССОВ В КОНТУРЕ ГАЗОВОЙ ТУРБИНЫ РЕАКТОРА TИПA SSTAR CO СВИНЦОВЫМ ТЕПЛОНОСИТЕЛЕМ*

\author{
() 2020 г. Чистов А.С. ${ }^{1}$, Савихин О.Г. ${ }^{2}$, Савихин И.О. ${ }^{3}$ \\ ${ }^{1}$ Научно-исследовательский институт механики \\ Национального исследовательского Нижегородского государственного \\ университета им. Н.И. Лобачевского, Нижний Новгород, Российская Федерация \\ ${ }^{2}$ Национальный исследовательский Нижегородский государственный \\ университет им. Н.И. Лобачевского, Нижний Новгород, Российская Федерация \\ ${ }^{3}$ СириусХМ Радио Инк, Дирфилд-Бич, Флорида, США
}

chistov@mech.unn.ru

Поступила в редакичию 04.09.2020

Рассмотрена задача математического и численного моделирования нестационарных процессов в замкнутой газотурбинной установке в составе блока преобразования энергии реактора со свинцовым теплоносителем. Задача решается в общей постановке, в которой газовый контур может включать в себя произвольное количество турбин с предварительным нагревом газа, произвольное число компрессоров с предварительным охлаждением газа и теплообменник регенеративного подогрева газа. Рассмотрены варианты одновальной и двухвальной газотурбинных установок. Используется точечная идеализация при моделировании проточной части турбины и компрессора.

Тепломассоперенос в контурах циркуляции свинцового и газового теплоносителей описан в одномерном приближении. Учитывается возможность взаимных фазовых превращений расплав-твердая фаза в свинцовом теплоносителе. Расчет тепломассопереноса в контурах осуществляется в рамках единого подхода, в котором циркуляционный контур представлен в виде набора взаимосвязанных теплогидравлических элементов (каналов). Интегрирование системы уравнений тепломассопереноса в контуре осуществляется с использованием быстродействующего алгоритма скалярной прогонки. В расчетном алгоритме предусмотрена возможность учета источников импульса и массы в произвольных узловых точках контура. Это дает возможность «сквозного» счета при интегрировании системы уравнений газовой динамики по замкнутому контуру с учетом изменения адиабатических перепадов давлений в точках расположения турбин и компрессоров на каждом временном шаге.

Рассмотрена возможность применения интегральной формы уравнения количества движения при моделировании тепломассопереноса в газовом контуре.

Для реактора типа SSTAR со свинцовым теплоносителем и газотурбинным циклом преобразования энергии выполнен расчет аварийного процесса

* Выполнено при финансовой поддержке РФФИ (гранты №o16-08-01114a, 19-08-01092a). 
с разрывом горячего газопровода. Получено, что, в отличие от реактора с паротурбинным циклом преобразования энергии типа БРЕСТ, затвердевание свинца в газонагревателях в данной аварии не возникает.

Результаты исследования могут быть использованы при разработке проектов реакторов со свинцовым теплоносителем и высокотемпературных газовых реакторов.

Ключевые слова: газотурбинная установка, газонагреватель, компрессор, тепломассоперенос, математическая модель, расчетный алгоритм, реактор, регенератор, свинцовый теплоноситель.

\section{Введение}

Статья посвящена разработке математического и программного обеспечения для исследования переходных и аварийных процессов в реакторных установках IV поколения на быстрых нейтронах со свинцовым жидкометаллическим теплоносителем - Lead-cooled Fast Reactor (LFR).

Проекты LFR активно разрабатываются во многих странах [1-8] и направлены, в первую очередь, на достижение качественно нового уровня безопасности реактоpa на основе принципа естественной самозащищенности. Под этим термином понимают способность реакторной установки (РУ) к автономному переходу (без участия активных средств управления и защиты) в подкритическое состояние и дальнейшему безопасному расхолаживанию (без превышения допустимых значений температур и давлений) при самых тяжелых авариях. Автономность процесса должна быть обеспечена внутренними обратными связями, действующими в реакторной установке, которые основаны на естественных свойствах ядерного топлива, теплоносителя и реализованных схемных решениях.

Разработка композиции и компоновки активной зоны и теплогидравлических схем циркуляции теплоносителя осуществляется на ранней стадии проектирования РУ. Параллельно проводится расчетный анализ самозащищенности РУ. Для этого оцениваются максимальные и асимптотические значения параметров в проектных и запроектных авариях. Для проведения таких исследований в распоряжении разработчиков должны быть средства компьютерного моделирования динамики РУ, которые, во-первых, должны адекватно отражать основные физические процессы и обратные связи в РУ, во-вторых, иметь гибкую открытую архитектуру, позволяющую легко отслеживать изменения, вносимые в проект. Такие программы могут быть основаны на упрощенном описании нейтронно-физических процессов в реакторе и теплогидравлических процессов в контурах циркуляции теплоносителей, а именно на точечной модели нейтронной кинетики и одномерном описании тепломассопереноса. В качестве примеров таких моделей и программ можно привести разработки [9-12]. Из них Relay-2 [9] фирмы General Atomics и симулятор на основе кода GTMHR [10] ОКБМ «Африкантов» предназначены для проектирования РУ с высокотемпературным газовым реактором, а программный комплекс (ПК) «Расплав» $[11,12]$ НИИ механики Нижегородского госуниверситета предназначен для моделирования нестационарных процессов в реакторах LFR.

С помощью ПК «Расплав» для РУ типа БРЕСТ был исследован специфический для реакторов LFR класс аварий с образованием твердой фазы в свинцовом теплоносителе [13]. БРЕСТ-ОД-300 - это реактор LFR с паротурбинным циклом преобразования энергии $[1,2]$. В [13] показано, что возможны сценарии с полным пере- 
крытием твердой фазой свинца проходных сечений парогенераторов, что приводит к катастрофическим последствиям. Исследование позволило сформулировать условия, выполнение которых исключает возникновение таких последствий.

Наряду с проектами LFR с паротурбинным циклом преобразования энергии существуют проекты LFR с газовыми турбинами $[4,14]$. Исследование класса аварий, представленное в [13], для LFR с газотурбинным циклом преобразования энергии является столь же актуальным. В связи с этим возникает задача разработки математического и алгоритмического обеспечения для ПК «Расплав», реализация которого позволит проводить указанные исследования.

Контур замкнутой газотурбинной установки (ЗГТУ) конструктивно существенно отличается от контура паротурбинной установки, поэтому моделирование нестационарных процессов в нем требует специального рассмотрения. Поскольку существует множество вариантов теплогидравлических схем ЗГТУ, задача математического и численного моделирования динамики ЗГТУ была сформулирована в достаточно общем виде. На основе разработанных моделей и расчетных алгоритмов создана расширенная версия ПК «Расплав», ориентированная на расчет динамики LFR как с паротурбинным, так и с газотурбинным циклом преобразования энергии.

Тестовые расчеты выполнены для LFR типа SSTAR (Small SecureTransportable Autonomous Reactor) [4] с газотурбинным циклом преобразования энергии, проект которого разрабатывается в кооперации тремя национальными лабораториями США (Argonne, Livermore, Los Alamos). В проекте двухконтурной реакторной установки SSTAR с номинальной мощностью 20 МВт продемонстрирована возможность достижения высокого КПД ( 44\%) при использовании ЗГТУ со сверхкритической двуокисью углерода.

\section{1. Математическая модель}

Общий подход. Математическое моделирование процесса взаимодействия потока газа с лопатками турбины представляет собой весьма сложную задачу, которая требует совместного решения уравнений трехмерной газовой динамики, движения ротора турбины и уравнений электродинамики электрогенератора. На практике для расчета параметров турбины в переходных процессах применяется зависимость Стодолы - Флюгеля [15]. Для дозвуковых сопел эта зависимость имеет вид

$$
\frac{G_{1 j}}{G_{1 j}^{*}}=\sqrt{\frac{P_{1 j}^{2}-P_{2 j}^{2}}{P_{1 j}^{* 2}-P_{2 j}^{* 2}} \cdot \frac{T_{1 j}^{*}}{T_{1 j}},}
$$

для сверхзвуковых сопел -

$$
\frac{G_{1 j}}{G_{1 j}^{*}}=\frac{P_{1 j}}{P_{1 j}^{*}} \sqrt{\frac{T_{1 j}^{*}}{T_{1 j}}},
$$

где $G, P, T$ - соответственно массовый расход, давление и абсолютная температура, а индексы $1 j, 2 j$ обозначают вход и выход $j$-й турбины (для ЗГТУ $G_{1 j}^{*}=G^{*}$ ). Индекс * здесь и далее обозначает стационарное значение параметра. Нестационарные процессы в проточной части турбины будем рассматривать в рамках идеализации (1), (1'). 
Рассмотрим в общем виде теплогидравлическую схему одновальной ЗГТУ, ко-

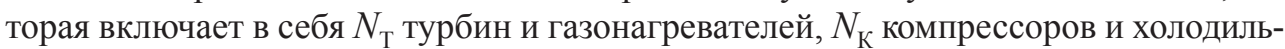
ников, а также регенератор (рис. 1). Система регулирования не рассматривается.

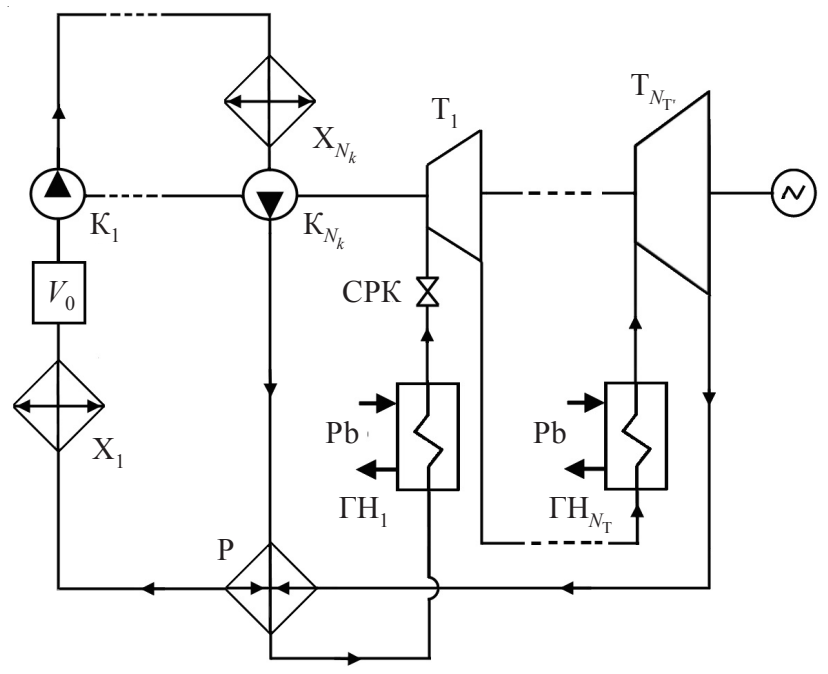

Рис. 1

В соответствии со схемой циркуляция газа в контуре осуществляется следующим образом. Газ проходит $N_{\text {T }}$ последовательных циклов нагрева в газонагревателях $\Gamma_{i}$ и совершения полезной работы на ступенях турбины $\mathrm{T}_{i}$. С выхода последней турбины газ поступает в регенератор Р. Регенератор - это теплообменник, в котором газ отдает оставшееся тепло встречному потоку газа, поступающему в первый контур с выхода последнего компрессора $\mathrm{K}_{N_{k}}$. Повышение давления в контуре осуществляется путем сжатия газа в компрессорах $\mathrm{K}_{j}$, перед поступлением в компрессор газ охлаждается в промежуточном холодильнике $\mathrm{X}_{j}$. Термодинамический цикл замыкается.

Для описания тепломассопереноса газа в контуре ЗГТУ воспользуемся одномерной системой уравнений газовой динамики, выбрав в качестве независимых переменных энтропию и давление $[16,17]$. В энергетическом балансе потока газа будем пренебрегать необратимыми потерями энергии за счет вязкого трения и теплообмена с окружающей средой по причине их малости по сравнению с тепловыми потоками в ГН и адиабатическими теплоперепадами. Потери давления на трение в контуре ЗГТУ будем учитывать только в газонагревателях и соединительных газопроводах. В проточных частях турбин и компрессоров гидравлическими потерями будем пренебрегать в силу их малости по сравнению с перепадами давлений в этих элементах контура.

Таким образом, процесс тепломассопереноса в контуре ЗГТУ за исключением участков активного теплообмена в газонагревателях можно считать изоэнтропийным. Кроме того, будем использовать сосредоточенную идеализацию турбины и компрессора, которая предполагает ступенчатое изменение давления в соответствующих точках контура. Тогда с учетом источников тепла и импульса система уравнений одномерного течения газа для канала с площадью сечения $F$ с учетом замыкающих соотношений может быть записана в виде: 


$$
\begin{gathered}
F \frac{\partial \rho}{\partial \tau}+\frac{\partial G}{\partial z}=0, \\
F \rho \frac{\partial s}{\partial \tau}+G \frac{\partial s}{\partial z}=\frac{q}{T}, \\
\frac{1}{F} \frac{\partial G}{\partial \tau}+\frac{1}{F^{2}} \frac{\partial G^{2} v}{\partial z}+\frac{\xi}{2 d_{\Gamma}} \frac{G^{2} v}{F^{2}}+g_{z} \rho+\frac{\partial P}{\partial z}=-\sum_{j} \Delta P_{j} \delta\left(z-z_{j}\right), \\
\rho=\rho(P, s), \quad T=T(P, s), \quad \Delta P_{j}=\Delta P_{j}(G, T), \\
q= \begin{cases}\Pi_{k} \alpha_{k}\left(T_{w}-T\right)_{k}, & z \in\left(z_{1 k}, z_{2 k}\right), \\
0, & z \notin\left(z_{1 k}, z_{2 k}\right),\end{cases} \\
\theta_{k} \frac{d T_{w k}}{d \tau}=\Pi_{k}\left[\widetilde{\alpha}_{k}\left(\widetilde{T}-T_{w}\right)_{k}-\alpha_{k}\left(T_{w}-T\right)_{k}\right], \\
\theta_{k}=(\rho C \Pi \delta)_{w k}, \quad \widetilde{T}=\widetilde{T}(\widetilde{z}, \tau) .
\end{gathered}
$$

Здесь обозначено: $\tau$ - время; $z$ - продольная координата; $\rho$ - плотность газа; $s-$ удельная энтропия; $q$ - тепловая мощность, передаваемая на участке канала единичной длины; $P$ - давление; $g_{z}$ - проекция ускорения свободного падения на направление движения; $\xi$ - коэффициент гидравлического сопротивления трения; $d_{\Gamma}-$ гидравлический диаметр; $\Delta P_{j}-$ перепад давления на $j$-м компрессоре или турбине; $\delta(z)$ - дельта-функция Дирака; $T$ - абсолютная температура; $v=1 / \rho$ - удельный объем газа; П - периметр поверхности теплообмена; $\alpha$ - коэффициент теплоотдачи; $C$ - удельная теплоемкость стали; $\delta$ - толщина стенки теплообменной трубы; $z_{1 k}, z_{2 k}-$ входная и выходная координаты $k$-го теплообменника. Нижние индексы обозначают: $w$ - принадлежность к стенке теплообменной трубы; $k$ - номер теплообменника (холодильника, газонагревателя, регенератора). Верхний индекс тильда обозначает принадлежность другому контуру $k$-го теплообменника.

Из уравнений сохранения массы и энергии для объема холодного газа на выходе холодильника $\mathrm{X}_{1}$, записанных в форме

$$
\begin{gathered}
\rho V_{0} \frac{d i_{0}}{d \tau}=G_{01}\left(i_{1}-i_{0}\right), \\
V_{0} \rho_{P}^{\prime} \frac{d P_{0}}{d \tau}=G_{01}-G_{02}-V_{0} \rho_{s}^{\prime} \frac{d i_{0}}{d \tau}, \\
i_{1}=i\left(P_{1}, s_{1}\right), \quad s_{0}=s\left(P_{0}, s_{0}\right),
\end{gathered}
$$

можно получить граничные условия для системы (2):

$$
P_{\text {en }}=P_{0}(\tau)+H_{1}(G, \omega), \quad s_{\text {en }}=s_{0}(\tau), \quad P_{\text {ex }}=P_{0}(\tau),
$$

где $H_{1}$ - напор компрессора $\mathrm{K}_{1} ; G_{01}, G_{02}$ - расходы газа на входе в газовый объем $V_{0}$ и выходе из него; $i_{1}-$ удельная энтальпия газа на выходе из холодильника $\mathrm{X}_{1}$.

Перепад давления на компрессоре $\mathrm{K}_{j}$ в (2) $\Delta P_{j}=-H_{j}(G, \omega)$ выражается через его напорную характеристику

$$
\frac{H_{j}(G, \omega)}{H_{j}^{*}}=h_{2 j} \bar{G}^{2}+h_{1 j} \bar{G} \bar{\omega}+h_{0 j} \bar{\omega}^{2}, \quad \bar{G}=\frac{G}{G^{*}}, \quad \bar{\omega}=\frac{\omega}{\omega^{*}},
$$

где $\omega$ - частота вращения вала компрессора; $h_{i j}$ - коэффициенты напорной характеристики. 
На рис. 2 показан характерный вид напорной характеристики компрессора [18].

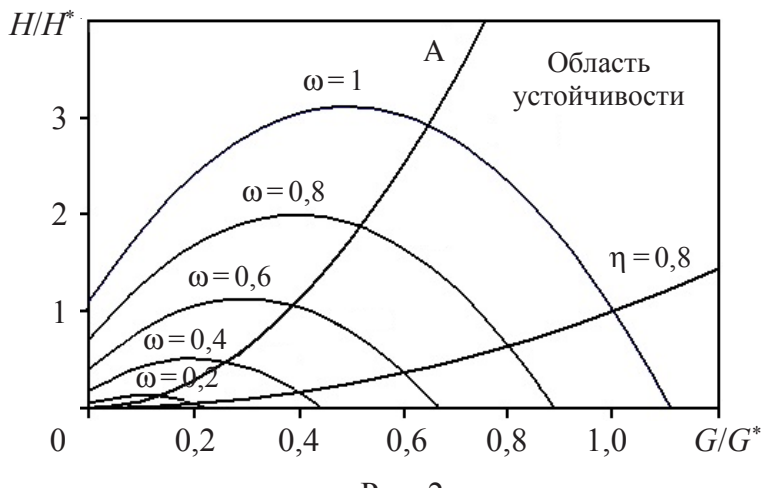

Рис. 2

Кривая А обозначает границу области устойчивых режимов, кривая $\eta=0,8-$ линия постоянного КПД.

В соответствии с (1), (1') перепад давления на турбине в (2) для дозвуковых сопел определяется зависимостью

$$
\Delta P_{j}(\tau)=P_{1 j}(\tau)-\sqrt{P_{1 j}^{2}(\tau)-\left(P_{1 j}^{* 2}-P_{2 j}^{* 2}\right) \frac{T_{1 j}(\tau)}{T_{1 j}^{*}} \frac{G_{1 j}^{2}(\tau)}{G^{* 2}}},
$$

для сверхзвуковых сопел в пренебрежении потерями давления в ГН - зависимостью

$$
\Delta P_{j}(\tau)=P_{1 j}(\tau)-P_{1 j+1}(\tau)=\frac{G_{1 j}(\tau)}{G^{*}} \sqrt{\frac{T_{1 j}(\tau)}{T_{1 j}^{*}}}-\frac{G_{1 j+1}(\tau)}{G^{*}} \sqrt{\frac{T_{1 j+1}(\tau)}{T_{1 j+1}^{*}}}
$$

Уравнение вращения ротора одновальной ЗГТУ имеет вид [18]

$$
J \frac{d \omega}{d t}=M_{\mathrm{T}}-M_{\mathrm{K}}-M_{\mathrm{E}}
$$

где $J$ - момент инерции ротора;

$$
\begin{aligned}
& M_{\mathrm{T}}=\frac{\eta_{\mathrm{T}}}{\omega} \sum_{j=1}^{N_{\mathrm{T}}}\left(\Delta I_{\mathrm{T} j} \cdot G_{\mathrm{T} j}\right)-\text { вращающий момент турбины; } \\
& M_{\mathrm{K}}=\frac{1}{\eta_{\mathrm{K}} \omega} \sum_{j=1}^{N_{\mathrm{K}}}\left(\Delta I_{\mathrm{Kj}} \cdot G_{\mathrm{Kj}}\right)-\text { момент сопротивления компрессора; } \\
& M_{\mathrm{E}}-\text { момент сопротивления электрогенератора. }
\end{aligned}
$$

Здесь $\Delta I$ - теплоперепад, $\eta$ - коэффициент полезного действия, относящиеся к $j$-й турбине (индекс Т) или компрессору (индекс К). В двухвальном варианте ЗГТУ вал компрессоров и вал турбогенератора разблокированы и имеют собственные приводные турбины. Уравнения для частот компрессоров и генератора следуют из (5) и достаточно очевидны.

Теплоперепады в (5) могут быть найдены либо с помощью уравнения состояния $i=i(P, s)$

$$
\Delta I_{\mathrm{K} j}=i\left(P_{1 j}+H_{j}, s_{1 j}\right)-i\left(P_{1 j}, s_{1 j}\right), \quad \Delta I_{\mathrm{T} j}=i\left(P_{1 j}, s_{1 j}\right)-i\left(P_{1 j}-\Delta P_{j}, s_{1 j}\right),
$$


либо непосредственно из (4), (4'). Действительно, в силу $T d s=d I-v d P$ и изоэнтропийности процессов расширения-сжатия газа в турбине и компрессоре, связь между теплоперепадом и перепадом давления может быть задана соотношением:

$$
\Delta I=\int_{z j-\varepsilon}^{z j+\varepsilon} d i=\int_{z j-\varepsilon}^{z j+\varepsilon} v(P, s) d P
$$

или приближенно

$$
\Delta I_{\mathrm{T} j} \approx \frac{1}{2}\left[v\left(P_{1 j}, s_{1, j}\right)+v\left(P_{2 j}, s_{2, j}\right)\right] \Delta P_{j}, \quad \Delta I_{\mathrm{Kj}} \approx \frac{1}{2}\left[v\left(P_{1 j}, s_{1, j}\right)+v\left(P_{2 j}, s_{2, j}\right)\right] H_{j}(G, \omega) .
$$

Температура свинцового теплоносителя $\widetilde{T}=\widetilde{T}(\widetilde{z}, \tau)$, которая используется для расчета теплового потока в ГН ${ }_{j}$ в (2), вычисляется с использованием модели первого контура, она подробно описана в $[11,12]$. Температуру охлаждающей воды в холодильниках будем считать постоянной $T_{\infty}^{*}$.

Частный случай. Для газовых турбин малой мощности, протяженность газового контура которых мала, в (2) можно перейти к интегральной форме уравнения количества движения. Если отнести все гидравлические потери на участок контура до первой турбины, то интеграл этого уравнения по замкнутому контуру длиной $L$ будет иметь вид:

$$
\frac{L}{F} \frac{d G}{d \tau}=P_{0}+\sum_{j=1}^{N_{K}} H_{j}(G, \omega)-P_{11}(\tau)-\frac{1}{2} \int_{0}^{L} \frac{\xi}{d_{\Gamma}} \frac{G^{2} v}{F^{2}} d z-\int_{0}^{L} \rho g_{z} d z,
$$

где в соответствии с (1), (1') начальное давление для до- или сверхзвукового сопла

$$
P_{11}(\tau)=\sqrt{P_{0}^{2}(\tau)+\frac{G^{2}(\tau)}{G^{* 2}} \sum_{j=1}^{N_{\mathrm{T}}}\left(P_{1 j}^{* 2}-P_{2 j}^{* 2}\right) \frac{T_{1 j}(\tau)}{T_{1 j}^{*}}} \text { или } P_{11}(\tau)=P_{1 j}^{*} \frac{G(\tau)}{G^{*}} \sqrt{\frac{T_{1 j}(\tau)}{T_{1 j}^{*}}} \text {. }
$$

Предполагая, что промежуточные холодильники обладают достаточным запасом теплообменной поверхности, которая позволяет обеспечить равенство температуры газа на выходе из холодильника температуре конечного поглотителя $T_{\infty}^{*}$, граничные условия (3) можно записать в более простом виде:

$$
P_{\text {en }}=P_{0}^{*}+H_{1}, \quad S_{\text {en }}=s\left(P_{0}^{*}, T_{\infty}^{*}\right), \quad P_{\text {en }}=P_{0}^{*} .
$$

Для расчетов переходных процессов используются уравнения состояния расплава свинца [19] и реального газа $\mathrm{CO}_{2}$ [20]. Расчет стационарного режима ЗГТУ осуществляется с помощью итерационного алгоритма, суть которого изложена в [21].

\section{2. Расчетная модель}

Расчетная модель ЗГТУ (2) строится в рамках тех подходов, которые реализованы в ПК «Расплав». Циркуляционный контур (рис. 3) в расчетной модели представлен в виде набора взаимосвязанных теплогидравлических элементов (каналов), которые обозначены номерами 1-15.

Расчет тепломассопереноса в канале осуществляется с использованием явнонеявной разностной схемы и быстродействующего алгоритма скалярной прогонки [22]. Число узловых точек по пространственной координате может быть произвольным. Последовательности каналов образуют замкнутый тракт циркуляции газа. 
На входе и выходе тракта задаются граничные условия (3) или (6). В расчетном алгоритме [22] предусмотрена возможность учета источников импульса (а также массы) в произвольных узловых точках контура. Это дает возможность «сквозного» счета при интегрировании системы (2) по замкнутому контуру с учетом адиабатических перепадов давлений в точках расположения турбин и компрессоров на каждом временном шаге.

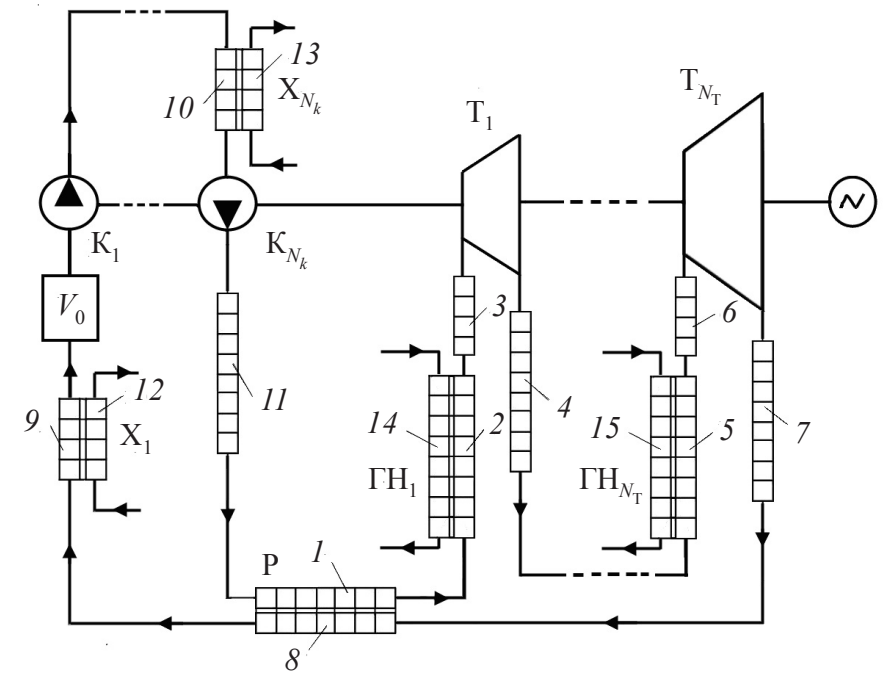

Рис. 3

Реализация расчетного алгоритма в ПК «Расплав» позволила провести численное моделирование динамики PУ типа SSTAR [4]. В качестве газотурбинной установки реактора SSTAR рассматривается вариант одновальной ЗГТУ с одной турбиной и четырьмя компрессорами: $N_{\mathrm{T}}=1$ и $N_{\mathrm{K}}=4$. Реакторная установка SSTAR содержит четыре одинаковых газонагревателя. В рассмотренном варианте схемы ЗГТУ все четыре ГН работают параллельно на одну турбину и, по сути, представляют собой четыре секции одного газонагревателя. $T-S$ диаграмма термодинамического цикла номинального рабочего режима ЗГТУ ( $T$ - температура, $s$ - удельная энтропия) показана на рис. 4. Основные данные номинального рабочего режима PУ SSTAR приведены в таблице 1.

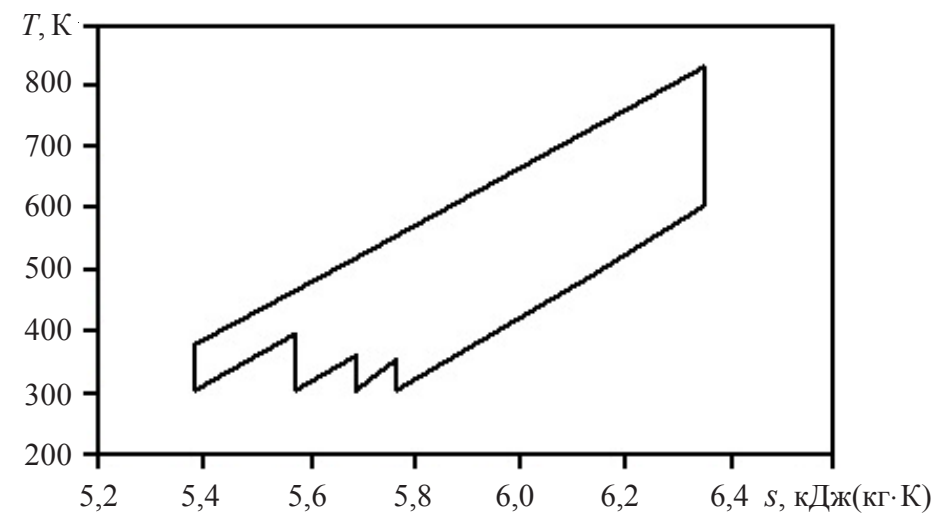

Рис. 4 
Исходные данные номинального рабочего режима PУ SSTAR

\begin{tabular}{|l|c|c|c|}
\hline $\begin{array}{c}\text { Элемент расчетной } \\
\text { схемы }\end{array}$ & $\begin{array}{c}\text { Давление/перепад, } \\
\text { МПа }\end{array}$ & $\begin{array}{c}\text { Температура, }{ }^{\circ} \mathrm{C} \\
\text { (вход/выход) }\end{array}$ & $\begin{array}{c}\text { Расход теплоносителя, } \\
\text { кг/с }\end{array}$ \\
\hline Регенератор & $1 / 0,05$ & $322 / 114,8$ & 170 \\
I контур & $16 / 0,001$ & $102,1 / 321$ & 170 \\
II контур & & & \\
\hline Газонагреватель: & $0,1 / 0,007$ & $563 / 420$ & 2168 \\
I контур & $16 / 0,005$ & $321 / 553,5$ & 170 \\
II контур & $16 / 14$ & $553,5 / 322$ & 170 \\
\hline Турбина & $2 / 0,001$ & $114,8 / 30$ & 170 \\
Холодильник 1 & $2 / 2$ & $30 / 68,5$ & 170 \\
Компрессор 1 & $4 / 0,001$ & $68,5 / 30$ & 170 \\
\hline Холодильник 2 & $4 / 3$ & $30 / 86,6$ & 170 \\
Компрессор 2 & $7 / 0,001$ & $86,6 / 30$ & 170 \\
\hline Холодильник 3 & $7 / 5$ & $30 / 122$ & 170 \\
Компрессор 3 & $12 / 0,001$ & $122 / 30$ & 170 \\
\hline Холодильник 4 & $12 / 4$ & $30 / 102,1$ & \\
Компрессор 4 & & & \\
\hline
\end{tabular}

\section{3. Расчет аварийного процесса}

На рис. 5-7 представлен расчет переходного процесса в ЗГТУ реактора типа SSTAR, вызванного разрывом газосборного коллектора на выходе ГН. Время разгерметизации принято равным 0,01с. Тестирование ПК «Расплав» при расчете разрыва трубопровода высокого давления проведено в [12] на экспериментальных данных [23]. Скорость звука двуокиси углерода в сечении разрыва составляет $300 \mathrm{~m} / \mathrm{c}$, и расход газа через ГН в разрыв (рис. 5) возрастает в первые сотые секунды до $\sim 1000 \%$. Далее за счет уменьшения давления происходит уменьшение расхода до полного опорожнения контура.

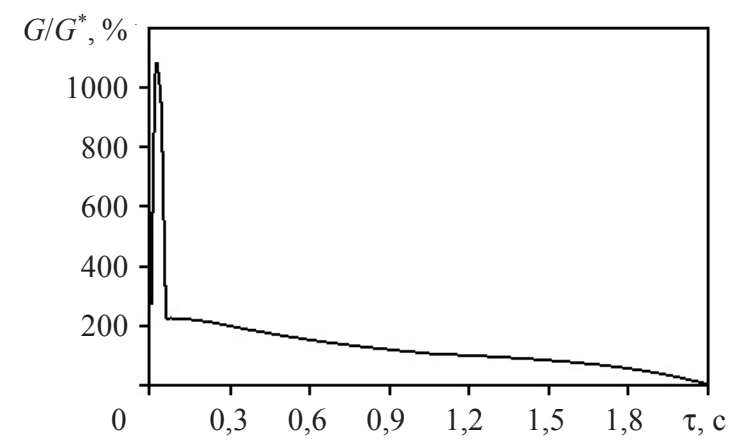

Рис. 5

При высоких скоростях газа происходит срыв компрессии. Физически это означает, что когда скорость газа существенно превышает скорость лопаток, сжатие газа не происходит и компрессор работает как гидравлическое сопротивление. В итоге давление на входе ГН (рис. 6, кривая 2) резко падает до значения давления холодного газа $\sim 3 \mathrm{MПа,} \mathrm{а} \mathrm{на} \mathrm{выходе} \mathrm{ГН} \mathrm{(рис.} 6$, кривая 1 ) - до атмосферного давления. После опорожнения контура на второй секунде давление в контуре становится равным атмосферному. 


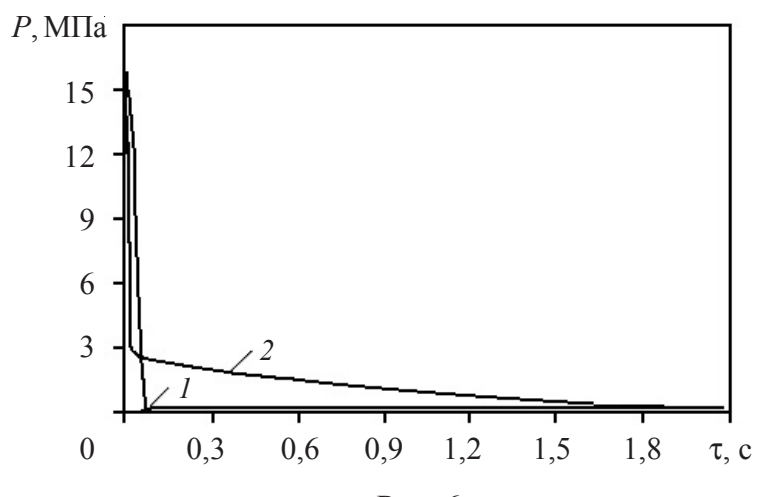

Рис. 6

Несмотря на то, что температура газа на входе в ГН резко уменьшается до значения температуры конечного поглотителя (рис. 7, кривая 3), температуры свинца в ГН за короткое время переходного процесса не успевают существенно измениться (рис. 7 , кривые 1,2 ). После опорожнения контура температуры в первом и во втором контурах ГН выравниваются (рис. 7, кривые 1,4). Таким образом, при разрыве горячего газопровода угрозы затвердевания свинца в первом контуре PУ SSTAR не возникает.

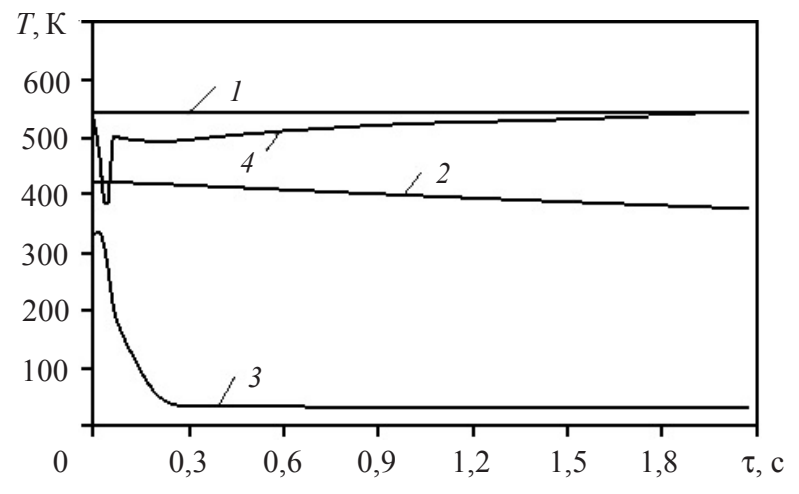

Рис. 7

Отметим, что в аналогичной аварии в РУ БРЕСТ с паротурбинным циклом преобразования энергии происходит полное перекрытие проходных сечений парогенераторов твердой фазой свинца. Однако вывод о том, исключена ли полностью возможность затвердевания свинца в ГН при газотурбинном цикле преобразования энергии, на основании данного расчета делать преждевременно. Необходимо провести полное исследование этого класса аварий, аналогичное [13].

\section{Заключение}

Разработаны математическая модель и алгоритм расчета нестационарных процессов в замкнутой газотурбинной установке, реализация которых в ПК «Расплав» позволила расширить его функциональные возможности.

С помощью расширенной версии ПК «Расплав» проведено численное моделирование аварийного процесса с разрывом горячего газопровода в LFR типа SSTAR с газотурбинной установкой. Получено что, в отличие от LFR типа БРЕСТ с паро- 
турбинной установкой, угрозы затвердевания свинцового теплоносителя не возникает.

В силу универсальности разработанных математической и расчетной моделей ЗГТУ они могут быть использованы при разработке проектов реакторов различного типа с газотурбинными установками.

\section{Список литературы}

1. Драгунов Ю.Г., Лемехов В.В., Смирнов В.С., Чернецов Н.Г. Технические решения и этапы разработки реакторной установки БРЕСТ-ОД-300. Атомная энергия. 2012. Т. 113. Вып. 1. С. 58-65.

2. Филин А.И., Цикунов В.С., Попов С.В., Нестеров Ю.В., Шварц А.Л., Колбасников А.В., Шмуклер Б.И., Гомболевский В.И., Радин Ю.А., Вахрушин М.П. Разработка схем, оборудования и режимов работы второго контура перспективной АЭС. Теплоэнергетика. 2001. №6. C. 27-31.

3. Liao J., Utley D. Study on reactor vessel air cooling for the westinghouse lead fast reactor. Nuclear Technology. 2020. Vol. 206. Iss. 2. P. 191-205. DOI: 10.1080/00295450.2019.1599614.

4. Smith C.F., Halsey W.G., Brown N.W., Sienicki J.J., Moisseytsev A., Wade D.C. SSTAR: the US lead-cooled fast reactor (LFR). Journal of Nuclear Materials. 2008. Vol. 376. Iss. 3. P. 255-259.

5. Alemberti A., Carlsson J., Malambu E. et al. European lead fast reactor - ELSY. Nuclear Engineering and Design. 2011. Vol. 241. Iss. 9. P. 3470-3480. DOI: 10.1016/j.nucengdes. 2011.03.029.

6. Alemberti A., Smirnov V. et al. Overview of lead-cooled fast reactor activities. Journal Progress in Nuclear Energy. 2014. Vol. 77. P. 300-307. DOI:10.1016/J.PNUCENE.2013.11.011.

7. Wu Y.C. Design and R\&D progress of China lead-based reactor for ADS research facility. Engineering. 2016. Vol. 2. Iss. 1. P. 124-131. DOI: 10.1016/J.ENG.2016.01.023.

8. Wu Y.C., Bai Y.Q. et al. Conceptual design of China lead-based research reactor CLEAR-I. Chinese Journal of Nuclear Science and Engineering. 2014. Iss. 2. P. 201-208.

9. Croft M.Z. Relay-2: The GT-HTGR transient performance analysis program. Report GA-A13880. General Atomic Company, 1976.

10. Митенков Ф.М., Кодочигов Н.Г., Болнов В.А., Богданова Е.В. и др. Разработка симулятора для проектирования реакторной установки ГТ-МГР. Вопросы атомной науки и техники. Сер. Физика ядерных реакторов. 2004. №3. С. 90-97.

11. Чистов А.С., Савихин О.Г., Овчинников В.Ф., Николаев М.Я. Математическая модель нестационарного тепломассопереноса в канале с жидкометаллическим теплоносителем с учетом процесса кристаллизации-плавления. Проблемы прочности и пластичности. 2016. T. 78. №4. C. 368-377. DOI: https://doi.org/10.32326/1814-9146-2016-78-4-368-377.

12. Чистов А.С., Савихин О.Г., Овчинников В.Ф., Кирюшина Е.В. Численное моделирование тепломассопереноса в парогенераторе реакторной установки типа БРЕСТ при возникновении кристаллизации свинцового теплоносителя. Проблемы прочности и пластичности. 2018. T. 80. №2. C. 267-280. DOI: https://doi.org/10.32326/1814-9146-2018-80-2-267-280.

13. Chistov A.S., Savikhin O.G., Ovchinnikov V.F., Kiryushina E.V. Numerical investigation of a class of accidents in the generation iv brest reactor involving the formation of a solid phase in the lead coolant. Thermal Engineering. 2019. Vol. 66. Iss. 8. P. 543-549. https://doi.org/10.1134/ S0040601519080019.

14. Петрушенко Ю.Я., Марченко Г.Н., Дружинин Г.И., Ильин В.К., Учарова А.У. Газотурбинная установка замкнутого цикла с ядерным реактором БРЕСТ. Изв. вузов. Проблемь энергетики. 2009. № 7-8. С. 111-118.

15. Турбины тепловых и атомных электрических станций. Под ред. А.Г. Костюка, В.В. Фролова. М.: Изд-во МЭИ, 2001. 488 с.

16. Рождественский Б.Л., Яненко Н.Н. Системы квазилинейных уравнений и их приложения к газовой динамике. М.: Наука, 1978. 592 с.

17. Сабаев Е.Ф. Системы сравнения для нелинейных дифференциальных уравнений и их приложения в динамике реакторов. М.: Атомиздат, 1980. 192 с. 
18. Селезнев К.П., Галеркин Ю.Б., Анисимов С.А., Митрофанов В.П., Подобуев Ю.С. Теория и расчет турбокомпрессоров. Л.: Машиностроение, 1986. 392 с.

19. Iida T., Guthrie R.I.L. The Physical Properties of Liquid Metals. Oxford, UK: Clarendon Press, 1988. 288 p.

20. Алтунин В.В. Теплофизические свойства двуокиси углерода. М.: Изд-во стандартов, $1975.546 \mathrm{c.}$

21. Михальцев В.Е., Панков О.М., Юношев В.Д. Регулирование и вспомогательные системы газотурбинных и комбинированных установок. М.: Машиностроение, 1982. 252 с.

22. Савихин О.Г. Динамика ЯЭУ с паровым энергетическим реактором: Дисс. ... канд. техн. наук. 05.14.03. Горький: НИИ механики при ГГУ им. Н.И. Лобачевского, 1990. 161 с.

23. Edwards A.R., O'Brien F.P. Studies of phenomena connected with the depressurization of water reactors. Journal of the British Nuclear Energy Society. 1970. Vol. 9. P. 125-135.

\section{References}

1. Dragunov Y.G., Lemekhov V.V., Smirnov V.S., Chernetsov N.G. Technical solutions and development stages for the BREST-OD-300 reactor unit. Atomic Energy. 2012. Vol. 113. Iss. 1. P. 70-77. DOI: $10.1007 / \mathrm{s} 10512-012-9597-3$.

2. Filin A.I., Cikunov V.S., Popov S.V., Nesterov Yu.V., Shvarc A.L., Kolbasnikov A.V., Shmukler B.I., Gombolevskij V.I., Radin YU.A., Vahrushin M.P. Razrabotka skhem, oborudovaniya i rezhimov raboty vtorogo kontura perspektivnoj AES [Development of thermal schemes, equipment and modes of operating regimes of the secondary circuit of a promising nuclear power plant]. Teploehnergetika [Thermal Engineering]. 2001. No 6. P. 27-31 (In Russian).

3. Liao J., Utley D. Study on reactor vessel air cooling for the westinghouse lead fast reactor. Nuclear Technology. 2020. Vol. 206. Iss. 2. P. 191-205. DOI: 10.1080/00295450.2019.1599614.

4. Smith C.F., Halsey W.G., Brown N.W., Sienicki J.J., Moisseytsev A., Wade D.C. SSTAR: the US lead-cooled fast reactor (LFR). J. Nucl. Mater. 2008. Vol. 376. Iss. 3. P. 255-259.

5. Alemberti A., Carlsson J., Malambu E. et al. European lead fast reactor - ELSY. Nuclear Engineering and Design. 2011. Vol. 241. Iss. 9. P. 3470-3480. DOI: 10.1016/j.nucengdes. 2011.03.029.

6. Alemberti A., Smirnov V. et al. Overview of lead-cooled fast reactor activities. Prog. Nucl. Energy. 2014. Vol. 77. P. 300-307.

7. Wu Y.C. Design and R\&D progress of China lead-based reactor for ADS research facility. Engineering. 2016. Vol. 2. Iss. 1. P. 124-131. DOI: 10.1016/J.ENG.2016.01.023.

8. Wu Y.C., Bai Y.Q. et al. Conceptual design of China lead-based research reactor CLEAR-I. Chinese Journal of Nuclear Science and Engineering. 2014. Iss. 2. P. 201-208.

9. Croft M.Z. Relay-2: The GT-HTGR Transient performance analysis program. Report GAA13880. General Atomic Company, 1976.

10. Mitencov F.M., Kodochigov N.G., Bolnov V.A., Bogdanova Ye.V. i dr. Razrabotka simulyatora dlya proektirovaniya reaktornoy ustanovki GT-MGR [Simulator development for GT-MGT reactor plant designing]. Voprosy atomnoy nauki i tekhniki. Ser. Fizika yadernykh reaktorov [Problems of Atomic Science and Technology. Series Mathematical Modeling of Physical Processes. Series Physics of Nuclear Reactors]. 2004. No 3. P. 90-97 (In Russian).

11. Chistov A.S., Savikhin O.G., Ovchinnikov V.F., Nikolaev M.Ya. Matematicheskaya model nestatsionarnogo teplomassoperenosa $\mathrm{v}$ kanale $\mathrm{s}$ zhidkometallicheskim teplonositelem $\mathrm{s}$ uchetom protsessa kristallizatsii-plavleniya [Mathematical model of non-stationary heat and mass transferin a channel with liquid-metal coolant takinginto account a crystallization-melting]. Problemy prochnosti i plastichnosti [Problems of Strength and Plasticity]. 2016. Vol. 78. No 4. P. 368-377 (In Russian). https://doi.org/10.32326/1814-9146-2016-78-4-368-377.

12. Chistov A.S., Savikhin O.G., Ovchinnikov V.F., Kiryushina Ye.V. Chislennoye modelirovaniye teplomassoperenosa $\mathrm{v}$ parogeneratore reaktornoy ustanovki tipa BREST pri vozniknovenii kristallizatsii svintsovogo teplonositelya [Numerical simulation of heat and mass transfer in the steam generator of the brest type reactor plant in the event of crystallization of lead]. Problemy prochnosti i plastichnosti [Problems of Strength and Plasticity]. 2018. Vol. 80. No 2. P. 267-280. DOI: https://doi.org/10.32326/1814-9146-2018-80-2-267-280. (In Russian). 
13. Chistov A.S., Savikhin O.G., Ovchinnikov V.F., Kiryushina E.V. Numerical investigation of a class of accidents in the generation iv brest reactor involving the formation of a solid phase in the lead coolant. Thermal Engineering. 2019. Vol. 66. No 8. P. 543-549.

14. Petrushenko J.J., Marchenko G.N., Druzhinin G.I., Il'in V.K., Ucharova A.U. Gazoturbinnaya ustanovka zamknutogo tsikla s yadernym reaktorom BREST [Gasturbine installation of the closed cycle with the nuclear reactor "Brest"]. Izvestiya vysshikh uchebnykh zavedeniy. Problemy energetiki [Power Engineering: Research, Equipment, Technology]. 2009. No 7-8. P. 111-118 (In Russian).

15. Turbiny teplovykh $i$ atomnykh elektricheskikh stantsiy [Turbines of Thermal and Nuclear Power Plants]. Eds. A.G. Kostyuk, V.V. Frolov. Moscow. MEI Publ. 2001. 488 p. (In Russian).

16. Rozhdestvenskiy B.L., Yanenko N.N. Sistemy kvazilineynykh uravneniy i ikh prilozheniya $k$ gazovoy dinamike [Systems of Quasilinear Equations and their Applications to Gas Dynamics]. Moscow. Nauka Publ. 1978. 592 p. (In Russian).

17. Sabayev Ye.F. Sistemy sravneniya dlya nelineynykh differentsialnykh uravneniy i ikh prilozheniya $v$ dinamike reaktorov [Comparison Systems for Nonlinear Differential Equations and their Applications in Reactor Dynamics]. Moscow. Atomizdat Publ. 1980. 192 p. (In Russian).

18. Seleznev K.P., Galerkin Yu.B., Anisimov S.A., Mitrofanov V.P., Podobuyev Yu.S. Teoriya $i$ raschet turbokompressorov [Theory and Calculation of Turbochargers]. Leningrad: Mashinostroyeniye Publ. 1986. 392 p. (In Russian).

19. Iida T., Guthrie R.I.L. The Physical Properties of Liquid Metals. Oxford, UK. Clarendon Press. 1988. 288 p.

20. Altunin V.V. Teplofizicheskiye svoystva dvuokisi ugleroda [Thermophysical Properties of Carbon Dioxide]. Moscow. Standards Publ. 1975. 546 p. (In Russian).

21. Mikhaltsev V.Ye., Pankov O.M., Yunoshev V.D. Regulirovaniye i vspomogatelnyye sistemy gazoturbinnykh i kombinirovannykh ustanovok [Regulation and Auxiliary Systems of Gas Turbine and Combined Installations]. Moscow. Mashinostroyeniye Publ. 1982. 252 p. (In Russian).

22. Savikhin O.G. Dinamika YAEU s parovym energeticheskim reaktorom [Dynamics of a nuclear power plant with a steam power reactor]. Diss. ... kand. tekhn. nauk. 05.14.03. [PHD thesis]. Gorky. Research institute of mechanics of Lobachevsky state university. 1990. 161 p. (In Russian).

23. Edwards A.R., O'Brien F.P. Studies of phenomena connected with the depressurization of water reactors. J. Brit. Nucl. Energy Soc. 1970. Vol. 9. P. 125-135.

\title{
NUMERICAL SIMULATION OF NON-STATIONARY PROCESSES IN THE GAS TURBINE CIRCUIT OF THE SSTAR LEAD-COOLED REACTOR
}

\author{
Chistov A.S. ${ }^{1}$, Savikhin O.G. ${ }^{2}$, Savikhin I.O. ${ }^{3}$ \\ ${ }^{1}$ Research Institute for Mechanics, National Research Lobachevsky State University \\ of Nizhny Novgorod, Nizhny Novgorod, Russian Federation \\ ${ }^{2}$ National Research Lobachevsky State University of Nizhny Novgorod, \\ Nizhny Novgorod, Russian Federation \\ ${ }^{3}$ SiriusXM Radio Inc, Deerfield Beach, Florida, USA
}

The problem of mathematical and numerical modeling of non-stationary processes in a closed gas turbine installation as part of an energy conversion unit of a lead-cooled reactor is considered. The problem is solved in a fairly General formulation, in which the gas circuit can include an arbitrary number of turbines with gas preheating, an arbitrary number of compressors with gas pre-cooling, as well as a heat exchanger for regenerative gas heating. Variants of both single-shaft and twoshaft gas turbine installations are considered. Point idealization is used when modeling the flow part of the turbine and compressor. Heat and mass transfer in the circuits of lead and gas coolants is described in a one-dimensional approximation. The possibility of mutual phase transformations of a melt-solid phase in the lead coolant is taken into account. Calculation of heat and mass transfer in the circuits is carried out within the single approach, in which the circulation circuit is represented 
as a set of interconnected heat-hydraulic elements (channels). Integration of the system of heat and mass transfer equations in the contour is performed using a fast scalar sweeping algorithm. The calculation algorithm provides the ability to take into account the sources of impulse and mass at arbitrary nodal points of the contour. This makes it possible to "end-to-end" computation when integrating the system of equations of gas dynamics along a closed loop, taking into account changes in adiabatic pressure drops at the points of turbines and compressors at each time step. The possibility of using the integral form of the momentum equation for modeling heat and mass transfer in a gas circuit is considered. For the SSTAR-type reactor with a lead coolant and a gas-turbine energy conversion cycle, the calculation of an emergency process with a rupture of a hot gas pipeline was performed. It was found that, in contrast to a reactor with a steam-turbine cycle of energy conversion of the BREST type, lead solidification in gas heaters does not occur in this accident. The study results can be used in elaborating the designs of lead cooled reactors and high-temperature gas reactors.

Keywords: gas turbine installation, gas heater, compressor, heat and mass transfer, mathematical model, computational algorithm, reactor, regenerator, lead coolant. 